tiert werden, we n n sine einzige Ver. wendungamöglichkeit in der Fär. berei li egt, denn durch Erteilung deg Färbeschutzes hat das Patentamt anerkannt, daB eine Erfindung auf dem Gebiete der Färberei gemacht ist, zu deren Begründung die in der Darstellung licgende Erfindung nicht herangezogen wurde*). Angenommen, A hütte z. B. das Verfahren zum Färben mit Thioindigo als erster angemeldet und patentiert erhalten, und in der Beschreibung hätte er die Darstellung des Farbstoffes beschrieb'n, hicrauf aber keinen Anspruch gerichtet. B hätte nun $\mathrm{n}$ a ch $\mathrm{A}$ diesellye Herstellung des Körpers unter Begründung der technisclien Verwertbarkeit einzig und allein durch die Färbeeigenschaften des l'roduktes angemeldet. D u reh d a v v r hor erteilte Färbepatent wären dann die pattent fähigen Nomentader Dar H tellungserfindung nicht vorweg genommen. Der Beweis für die Richtigkeit dicser Auffassung liegt wohl darin, daB gegen die Patentierung irgendeines reuen Darstellungsverfahrens von Thioindigo, Gas von dem in der früheren Färbeanmeldung brschriebenen verschieden ist, patentrechtlich nicht das geringste einzuwenden wäre, auch wenn Thioindigo nur zum Färben verwendet werden könnte und dies den A schon geschützt wäre. I)ie alleinige Anmeldung des Fürbeverfahrens hat also cine gewisse Gefahr für den lirfinder.

[A. 46.]

\section{Fortschritte in der Chemie der Gärungsgewerbe im Jahre 1911.}

\section{Von O. Monn.}

(Éingeg. 4.14. 191:...)

I. Chemic der Rohstoffe.

Dic Ger 8 te $n$ des Berichtajahres sind durchweg stickstoffarm, sie zeigen sich aber wenig lagerfest und machen bri der Verarbeitung Sehwierigkeiten. Win $(\mathrm{l}$ is $(\mathrm{h}$ 1) bezcichnet sie als ,unterreif", er ist der Ansicht, daB infolgedessen beim Lagern Nachreifungsvorgänge einsetz'n, welche die geringe Lagerfestigkeit verursachen. Um eine Gerste im Hinblick a u die zu erwar. tonde Extraktausbute aus dem $M$ a $l$ z $z$ be werte $n$, stehen verschiedene Methoden zur Verfügung: Sortierung und Stickstoffbestimmung, Gerstenextraktbestimmung, Stärke-

4) Das Patentamt hätte vielleicht in diesem Fall ein Färbepatent nicht erteilen, sondern den Anmelder des Färbeverfahrens darauf hinweisen sollen, daß die unentbehrliche Voraussetzung für dieses die Darstellung des Produktes sei, und das Färbeverfahren allein gar keine selbständige Bedeutung habe, der wesentlichste und allein patentfähige Teil seiner Erfindung also das Darstellungsverfahren sei. Für dio Beurteilung der nach Bekanntmachung oder Erteilung des l'atentes auf das Färbeverfahren zur patentamtlichen Behand. lung kommenden Darstellungsanmeldung kommt die Frage, ob das Färbeverfahren zu Recht oder Unrecht geschützt wurde, nicht mehr in Betracht.

1) Wochenschr. f. Brauerei 29, 160. bestimmung. Nach C. J. Lintner ${ }^{2}$ ) ist die letztgenannte Methode für den gedachten Zweck die empfehlenswerteste. E. Moufang und A. Schee ${ }^{3}$ ) wollen auch den $H$ opfen durch Extrakt be s t m m ung bonitieren. Der Hopfen wird unter bestimmten Versuchsbedingungen eingemaischt und in der erhaltenen Maische der Extrakt bestimmt. Nach der Vff. Beobachtungen zeigen die besten Hopfensorten die größte Extraktergiebigkeit. Zur Extraktion des Hopfens bei Be stim $m$ ung der $\alpha$ - und $\beta$. Bittersäure wird, wie G. Fe ue r s te in 4) beobachtet hat, zweckmäBig Petroläther vom Siedepunkt $45-50^{\circ}$ verwendet. Niedriger siedender extrahiert nicht vollständig. Vf. empfichlt., die Höhe der Hopfengale im Sudhaus dem Bitterstoffgehalt des Hopfens anzupassen. Ias $\mathrm{Humulen}$ des $\mathrm{Hop} \mathrm{fe}^{2} \mathrm{n}$ bli

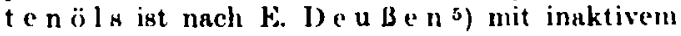
a-Karyophyllen identisch. Der ungew wihnlich trokkene Sommer des Jahres 1911 ist Crsache, daB dic $\mathrm{K}$ art of f e In viclfach eine ungewöhnliche $\mathrm{Zu}$ sammensetzung zeigen; Trockensubstany und Stärkegehalt erreichen eine auberordentliche Höle,

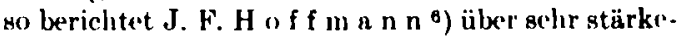
reiche Kartoffeln, die bis zu $34^{\circ} \%$ Stärke enthiclten.

Bei dem sehr niedrigen Ernteertag an Kartoffeln sind cinige Arbeiten über anderweitige Rohstoffe von Interesse, deren Verarbeitung auf Spiritus möglich ist. So zeigt $\left(\mathrm{G} . \mathrm{F} \circ \mathrm{th} \mathrm{h}^{\mathrm{T}}\right.$ ), daß unter Umständen P'ülpe mit wirtschaftlichem Erfolg auf Spiritus verarbeitet werden kann. Zi cho rienwurzel ergibt nach W. Donselt ${ }^{8}$ ) nuf $100 \mathrm{~kg}$ nur cinen Spiritusertrag von 4,41 reinen $\mathrm{Al}$ kohols, die Verarbeitung ist also nicht lohnend. Koloczok Anta (日) schildert die zwockmäBigste Verarbeitung von $Z$ u c k e r r ü be n nuf Spiritus, nach seinen Berechnungen ist der Rübenspiritus billiger als Kartoffelspiritus.

II. Verarbeitungder Rolstoffe. (Mälzen und Malz, Maische- und Würzebereitung.)

In einer Arbeit, betitelt: $S t u d i e n$ ï ber das Ein we ic he $n$, zejgt $O$. Fürn roh ${ }^{10}$ ). daß sich die Wirkung des Einweichens bis auf das fertige Bier erstreckt. Namentlich die Limwandlung der Eiweißstoffe wird durch die Einweichtempern. tur beeinflußt. Höhere Einweichtemperatur erhöht die Meng: des durch Phosphorwolframsüure fällbaren Stickstoffs, dafür geht die Menge des durch Zinksulfat fällbaren Stickstoffs zurück. W. W i n disch ${ }^{11}$ ) hat Weich versuche mit an ges äuertem $W$ asse $r$ angestellt in der $A b$. sicht, die Aciditätsabnahme $z u$ verhinclern, wie sic bei der Kalkwasserweiche zu beobachten ist. Bei gesunden Gersten wurden zunächst. im Iabora(1911)

2) Z. ges. Brauwesen 34, 301; diese Z. 24, 1561

3) Wochenschr. f. Brauerei 28, 449; diese Z. 25. $184(1912)$.

4) Ibid. 28, 169; diese Z. 24, 1292 (1911).

5) J. prakt. Chem. 83, 483.

6) Z. f. Spiritus-Ind. 34, 522.

7) 7. f. Spiritus-Ind. 34, 25.

8) lbid. 34, 93: diese Z. 24. 1052 (1911).

9) 1bid. 34, 239, 252; diese Z. 24, 1548 (1911). (1912)

10) Z. ges. Brauwesen 34, 449; diewe Z. 25. 183

11) Jahrb. Vers. u. Lehranst. f. Brauerei 14, 38. 
torium günstige Ergebnisse erzielt, во da $B$ nun Versuche in der Praxis angestellt werden sollen. Nach C. Winckler ${ }^{12}$ ) übt gute Lüftung des Weichwasers cine Beschleunigung a $u$ f $d$ i $K$ e i m u $n$ ans. Ws handelt sich hierbei zweifelios um eine Saucrstoffwirkung, denn diese Beschleunigung wird besonders auffällig bei Zusatz von Wasserstoffsuperoxyd zum Weichwasger. Hei gecigneter Konzentration des Weichwassers an diese'm Stuffe spitzt dic Gerste schon nach 24 Stdn. lie Wurzelkeime bleiben wehr klein, während die Blattkeime sehr kräftige Entwicklung zeigen. I. O. $\mathrm{t}$ t inger und F. H i $\mathrm{s}$ c h 13) haben sich ein Verfuhren zur Verminderung derstick. stoffgehaltes und Erhïhung dor Fxt rakta us be u te bei Braugerste während des Weichens patentieren lassen. Das Verfaliren best ht darin, daB ein elekt rischer ftrom von hoher Stromdichte durch dir Flïssigkeit genchickt wird, in der sich die Gerste suspendiert befindet. $C$. $\mathrm{Bl}$ lo is ch beschreibe ein meues, von $\mathrm{K} r o p$ f 14 ) angkgelenes II älzungusystam. Die Kej. mung erfolgt in Keimkïsten, in denen das Malz. sehr hoch gexchichtet wird, und wobei die Luftza. fulit awecks starker Kohlensäureansammlung im Koimgut zeitweise abgesperrt wird. Der Erfolg des Arlwitens nach $\mathrm{K}$ r o $p$ f $f$ ist ein sehr geringer Malzwchwand, weil die timung infolge Siluerstoffman. kels wturk yehemmt wird.

Eine Arbeit von J. Wa instain 15) betrifft die Kongulationsverhältaniage der lowlichen EiweiBstoffedes $M a l z e s$ und die Einwirkungderproteolyti. schen En\%ymo a uf das koagulier. ba re Eiweib. Fon den Ergebnissen der Arbeit mögen folgende erwähnt sein: Die Optimultempe. ratur der proteolytischen Malzenzyme liegt bei $\mathbf{5 0}$ bis $75^{\circ}$. Fin aus hellem Malz hergestellter Kaltwaserauszug beginnt bei $5 \cdot 2-54^{\circ}$ zu koagulieren, bei Auszügen aus dunklem Malz war dieser Punkt nicht so scharf bestimmt. Nach dem KongreB. mairchverfahren bereitrote Würzen zeigen Koagulierung bei $85^{\circ}$.

Kiyohisa Yunhimura 16) hat aus In lykeimen Histidin. Cholin und Betain isolieren können, nicht dugegen Argirin, Vernin und Aspuragin. Von Zuckern fand er Maltose und Invertzucker, aber keinen Inosit. In Fortsetzung früherer Arbeiten 17) üler M a l z dias t a s e kommt T. ('hrzas z c z 18) zur Annalune, duB die stark verflïssigende und die verzuckernde Wirkung durch versehiedene Enzyme hervorgerufen wird. Durch fraktionierte Fällung von Mnlzauszügen mit Amnoniumsulfat gelang $a$ dem Vf., Niederschläge zu crhulten, deren erstu. Fraktion stärker verflüssi. gend, deren letzte Fraktion stärker verzuckernd wirkte.

Die II aize aus den laller Gersten

12) Brasserie et Malterie 1911, 228; nach Wochenschr, f. Brauerei 28, 497.

13) D. R. P. 235877 .

14) Z. ges. Brauweren 34, 209; diese Z. 24, 1291 (1911).

1.5) Ibid. 34, 308; diese Z. 24, 1985 (1911)

10) Biochem. Z. 31, 221

17) Diese Z. 24, 775 (1911).

1*) Wochenschr. f. Briuerei z8, 510. sind, entsprechend dem niedrigen EiweiBgehalt der Gersten, eiweißarm und extraktreich, wie aus zahlreichen analytischen Daten hervorgeht, die $\mathbf{H}$. $K$ e i l und $E$. Weber ${ }^{19}$ ) veröffentlichten. In einer Arbeit, betitelt: $M$ echan is che und therminche Finflüsncauf die Höhe der Wasser- und Exiraktzahlen wertedes M alzos, macht A. W lokk a ${ }^{20}$ ) Vorschläge für Vereinbarungen, betreffend dic Me. thoden bei der Malzanalyso zwecks Erziclung ülx.r. cinstimmender Analysenergebnisse. (r. J a $k a b$, 21) zeigt, daB bei f a $r$ bm a I zeine Wert vermin. derung dureh hohen Wh a a regalt und geringe betraktaumbute we niger von Belang ist, Hls genügend bohe's Färlever mögen und Geschmacksreinheit.

Eine große Zahl von Arbeiten behandelt die

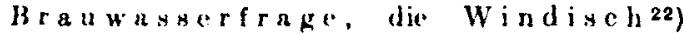
zum Gegenstand cines umfangreichen Vortrages macht. He zeigt darin, dab der Gehalt an carbonuten fïr die Beurteilung des Wassers von beson. derer Hedeutung ist, die C'arbonate ülx'n einen ungünstigen binflub nuf Verzuckerung, Ausbeute. Farbe, Vergürung aus, weil wise die Acidität der Würze herabdrücken. 1)a nur gelöstes, nicht abor fest ausgerschiedenes (alciumcarbonat diese un. günstigen Wirkungen hat, ist e's nach F. Pot t ${ }^{23}$ ) wichtig, festrustellen, welche Mengen ('arbomat durch Erwärmen bis $100^{\circ}$ nbscheidbar sind. ('alciunsulfat soll diese Abscheidung günstip becinflussen. Auch $W$ ind $i$ s $c h$ und seine Mitarleiter ${ }^{24}$ ) bestätigen, daB nur gelïstes Carbonat ungünstig wirkt, so daß nach dem Fntcarbonisieren des Was. wers durch Kochen Filtration nicht erforderlich ist. Enerwünscht grober Gipggehalt lüßt sich durch Kochen mit Bariuncarbonat beseitizen, Wie eben. falls $W . W$ ind is $c h{ }^{25}$ ) durch Versuche festatellt, gibt dic leseitigung der Carbonate durch Neutralisation mit Säuren (Schwefelsäure, Salzsäure, l'hosphorsäure) keine ganz zufricdenstellenden Frgebnisac. Wine weitgehende Entcarbonisierung erzielt E. I $a$ । 0 we $z^{26}$ ) dadurch, daB er das Wasser in einem geschlossenen, mit guter Rührvorrichtung versehenen Gefäb hoch orhitat. Die Curbonute scheiden sich bei dieser Pehandlung in fein kryatal liner Form aus. Versuche in der Praxis ergeben nach W. Koch'it z e $r^{27}$ ) nit derartig entcarbunisiertem" Wasser gute Firfolge. Fine längere Arleit von $O$. II i $k$ o v 8 k ${ }^{28}$ ) behandelt den Einflub des Brauwassers a u d ie Eigensch a tendes 1 ieres. Vf. arbeitet mit künstlieh bereiteten Wüssern und studiert den Einflul verschiedener Calcium- und Magnegiumsalze auf Verzuckerung, Farbe der Würze, Schaum. haltigkeit, Stickstoffgehalt, Aschegehalt usw. W.

19) Ibid. 28, 549

20) Ibid. 28, 317; diese Z. 24, 1405 (1911).

21) Ibid. 28, 341; diese Z. 24, 2130 (1911).

22) Jahrb. Vers. u. Lehranst. f. Brauerei 14, 361.

23) Wochenschr. f. Brauterei 28,248 ; diese $Z$.

24, 1984 (1911)

24) Jahrb. Vers. u. Lehranst. f. Brauerei 14, 52.

26) Ibid. 14, 39.

26) Z. ges. Brauwesen 34, 509; Wochenschr. f. Brauerei 28, 494; diese Z. 25. 182.

27) Z. ges. Brauwesen 34, 574.

28) Ibid. 34, 49, 525; diese Z. 24, 1546 (1911) 
W in d i s $\mathrm{h}^{29}$ ) hat in Laboratoriumsversuchen zwecks Erhöhung der Würzeacidi . $t$ ä $t$ die noch unverzuckerten Maischen in it $t$ e 18 Bacillus Delbrücki gesäuert. Bei dreistündiger Säucrungszeit ließ sich so viel Milchsäure erzielen, daB etwa $65 \mathrm{~g}$ Calciumcarbonat auf 1 hl Maischwasser neutralisiert wurden. Die praktische Durchführbarkeit des Verfahrens ist noch festzuste?len.

Inkritimehen Betrachtungenzur Frage dersudha s a a $_{\text {s }}$ beute und zum Stsmminirzegehalt des Bieres erörtert $S$ tock mejer ${ }^{30}$ ) die Fehlerquellen, auf welche bei Berechnung der Ausbeute Rücksicht zu nehmen ist. Eine schr wesentliche kann in einem gröBeren Salzgehalt de's verwendeten Brauwassers liegen. Auch die Stammwärzenlerechnung aus der Bierzusam. mensetzung ist mit Unsicherheiten behaftet (siehe auch Gärungserz:ugnisse). C. Ble i $\mathrm{ch}$ und H. Leberle ${ }^{31}$ ) haben Versuche angestellt über Ausbeuten von II lacenaus derselbe $\mathrm{n}$ Gers te mit verschieden langer Tennenzeit unter Berücksichtung der Feinheit des Schrotes und verschiedener Maischverfahren. Die größte Ausbeute wurde nicht $n \mathrm{~m}$ Schlu $B$ der iiblichen Ten. nenzeit, sondern einen 'Tag vorher erhalten. Kürzere Führung verringert die Ausbeute, Verwendung von Feinschrot ist b'i solchen Malzen ein Mittel zur Stcigerung der Extraktergicbigkeit. Das Druckverfahren gibt auch bei Kurzmalzen sehr hohe Ausbeute. Ein $Z$ un a ischen von Caramel. m a l z empfiehlt $F^{*}$. S ch oe n f eld ${ }^{32}$ ) für Biere, die dunkel in der Furbe, röstaromatisch bitter im Geschmack sein sollen. Das Zumaischen von solchem Malz erhöht den I)xtringehalt der Maischen.

Die Extraktbestimmung in Wür. zen mittels Saccliarometer hat gegen. über der pyknometrischen Extraktbestimmung zu hohe Werte crgeben. (). Mo h $\mathrm{r}^{33}$ ) zeigt, daB die Ursache hierfür in der Nichtberücksichtigung der Verschiedenheit der Oberflächenspannung von Würze und Zuckerlösungen bei Einstellung der Saccharometer liegt. J. S a $t$ a $v$ a ${ }^{34}$ ) veröffent. licht einen längeren Aufrat\% im gleichen Sinne.

Die Säurebostimmung in Würze und Bier empfiehlt E. If o u f $n \| \mathrm{g}^{35}$ ) durch direkte Titration der ev. verd. Flüssigkeiten unter Anwendung von Phenolphthalein vorzunehmen, die erhaltenen Werte sind anzugeben in Gramm Milehsäure auf $1 \mathrm{hl} 10$ "iger Würzc. Klärung trüber Würzen mit Asbest ist nicht zulässig. Boi Bier ist die Kohlensäure durch Kochen völlig zu entfernen. F. Barn\&te in ${ }^{36}$ ) berichtet über die $\mathrm{Z}$ u s a m. mensetzung von I) ruektrebern. Durch Behandeln der Treber eine halbe Stunde lang bei

29) Jahrb. Vers, u. Lehranst. f. Brauerei 14, 62. (1912).

so) Z. ges. Brauweren 34, 473; diese $Z$. 25, 184

31) Z. ges. Brauwesen 34, 485 .

32) Wochenschr. f. Brauerei 28,371 ; diese $Z$.

24, 2130 (1911)

33) Ibid. 28, 304

34) Z. ges. Brauwesen 34, 398; diese Z. 24, 2131 (1911).

35) Wochenschr. f. Brauerei 28, 329; diese $Z$.

24, 2131 (1911).

36) Z. gex. Brauwesen 34. 54.
$3 \mathrm{Atm} .\left(144^{\circ}\right)$ wird die Stärke ganz zun Verschwinden gebracht, der Gehalt an wasserlöslichen Extraktstoffen ist um ca. $2 \frac{1}{2} \%$ zurückgegangen. Die Ver. daulichkeit der Eiweißstoffe ist herabgesetzt. (:. Kreuzer ${ }^{37}$ ) gibt ein jod titrimetrisches Verfahron zur Bestimming der $S t$ iirke in Trebern an.

Einige Artikel von (G. Foth $\left.{ }^{38}\right)^{30}$ ) belutndeln die neue Betriebswoise in den Brennereien, wie sie durch die Neuordnung der Branntweinsteuergesetzgebung bedingt wird. An Stelle der Dickmajschen mind Dïnnmaischen getreten, zu deren Bereitung geringers Malzgaben ausreichen. Dic Entzchalung ist überflüissig geworden, es genügt, die Maische zur Finternung gröberer Stïcke durch ein Sieb zu gieß"n. Die Hefoführung ist im allgemeinen die alte ge. blieben, bei den dünneren Maischen ist xorgfältige Reinhaltung der Hefe nötig. Zur möglichsten binschränkung der Alkoholverdunstungsverluste ('mp)pfiehlt nich, die Bottiche ledeckt zu halten, ev. die Kohlensäure zu waschen. Ije Ausleuten sind leessere als früher, sie betragen 66 - 67 I reinen Alkohol auf $100 \mathrm{~kg}$ eingemajschte Stärke.

$$
\begin{aligned}
& \text { III. (ï̈rungsorganismen und (i i - } \\
& \text { rungsvorgang. }
\end{aligned}
$$

F. $H$ a y d u ck und (i. A n d e r ${ }^{40}$ ) halxoll das bereits früher Ix-hundelte Themn des $\mathrm{E}$ in flusoce der Hefenaussant auf dia. Sprob bild un $\mathrm{k}$ von neuem experimentell $\mathrm{lx}$ handelt. Fs zeigt sich, daß auch günstige Wachstumsbedingungen nicht junstande sind, bei steigender Hefenussat die Ernte aus einem gegebenen Gärflüssigkeitsvolumen zu steigern. J)ie Versuche der Vff. lassen es wahrscheinlich erseheine'n, daB dic Ursache dieser brsoheinung Raummang(el, nicht Hemmung durch dic Gärungserzeugnisse ist.

Eine umfangreiche Arbeit über die $B$ ed c u tung des $Z$ ü cht ungsverfahrens für die chemisehe und physiologische 13 es chaf fen he it der Hefejveröfentlichen F. Schönfeld und H. Kra mp f ${ }^{41}$ ). Mit Va. riierung des Züchtungsverfahrens ließ sich Fiwerib-, Aschegehalt, Gehalt an Glykogen, Gehalt un Ihos. phorsäure und Verteilung dieger an löslich orguninche Verbindungen und lösliche anorganische Verbindungen variieren. Eine variierbure physikalische Figenschaft ist das spezifische (rewicht. In physiologiacher Beziehung licßen sich Interwchiede crziclen in Flokkungsvermögen, Triebkraft, Gärkraft, IViderstandsfähigkeit gegen höhere Temperatur. Hine Zusammenstellung der kigkebnisse früherer Arbeiten ïlx'r das Verhalten der Hafe in der praxis in Bazehung u ihren chemischen und bhysiologischen Figengehuf. te $n$ geben $F$. S chönfeld und $W$. H i r t42).

Nach Brobachtungen von $H$. $W$ j]l la) ijber die Leben sd a uer von Hefen in (iolu.

37) Ibid. 34, 277; diese Z. 24, 1985 (1911).

38) Chem.-Ztg. 35, 297; diese Z. 24, 1548 (1911).

39) Z. Spiritus-Ind. 34, 1.

40) Wochenschr. f. Brauerei 28. 233; diese $Z$. 24, 1986 (1811).

41) I bid. 28, 157; diene Z. 24, 1292 (1911)

42) lbid. 28,421 .

43) Zentralbl. Bakt.- u. Parasitenk. 11, 31, 436. 
t i ne $\mathrm{k}$ u I t u re n ist eine Würzegelatine nit $10^{\circ} \%$ Gelatine am günstigsten für die längere Frhaltung. Erforderlich ist Auflewahren der Kulturen in kühler, feuchter Luft.

F. Fh r li $\mathrm{ch}^{44}$ ) hat zu ermitteln versucht, welche Aruinosäuren oder Zuckerzerfallsprodukte von Hefen und Sehimmelpilzen zur Bild ung von Pl $\Omega$ s m a $i w e$ i $B$ ausgenutzt werclen können. Vf. benutzt in erster Linie Tyrosin als Stickstoffquelle, Milehwäure, Weinsäure, Amejsensüure, Glycerin und Ithylalkohol als Kohlenstoffquelle. Willia anomala kann bei Tyrosingegenwart sehr einfache Stoffe, wie Methyl- und Athylalkohol, zur biweißsynthese vorwerten, ähnliche Verhältnisse zeigt Oidium lactis. Kulturhefen und Kahmhefen können Brenztraubensüure schr gut sowohl als Kohlenstoffquelle als auch als Energie. quelle ausnutzen. Möglicherweise tritt diese Säure als Zwischenprodukt bei der alkoholischen Gürung auf. Der Bestätigung bedarf wohl noch eine Beobachtung $C h$. B. L i p m a n ${ }^{45}$ ), dahingehend, daB die Hefen die Fähigkeit lexitzen, Atmosphärstick. stoff $\mathrm{zu}$ ussimilieren.

P. I. ind ne ${ }^{40}$ ) hat, in Fortsetzung früherer Arlecitent7) eine grobe Zahl $H$ efen a uf ihr (i ärverm öge n gegenüber einer Anzahl Kohlehydrate, neben gewöhnlichen auch Inulin, Mannose, (inlaktose, Trehalose, a-Bethylglucosid, Arabinose, Rhamnose, Xylose untersucht. In nicht seltenen Füllen, in dene'n anfänglich keine Vergärung zu beobachten war, trat solche bei Verlängerung der (iärzeit noch ein. Ierselbe Forscher hat gemeinsam mit $O$. 11 o h $\left.r^{48}\right)$ eine Reihe von $D$ e $x$. trinen, Süuredextrin, Bier-und W ï r \% edextrin, auf ihre Vergärbarkeit durch Hefen und Schimnolpilze geprït. Ein Würzedex. trin wurde von den moisten Hefen etwas angegrif. fen, gegenülo'r don anderen Dextrinon zeigten die Kulturhefen meist kein Gärvormögen, wirksamer waren wilde Hefen, Tropenhefen und vor allem Monilis, Amylomyces- und Sachsiarten.

Das Verhalten abgepreBter Hefen beidersehlag probrkann nach W. Hen neber $g^{40}$ ) zur Erkennung des physiologischon Zustandes der Hefe dienen. Normale Hefe mit festem l'lasma hält dic Schlagprobe aus. Sohr schnell gewachsene Hofe dugegen besitzt weiches, reizbares Plasma, das I'rsuche des Naßwerdens der Hofe bei der schlagprobe ist. Eino Reihe von Arbeiten hundelt von $\operatorname{dem} \mathrm{E}$ influB des Waschens der Hofemit verd. Säuren. S) beobachtet $W$. Wind is c h ${ }^{60}$ ), daB Hefe, dic b.i sehr carbonatreichem Brauwasser zu stark Bruch bildete und daher nicht genügend weit vergor, sich clurch Waschen nit verd. Süuren erfolgreich behundeln ließ. Ahnlich günstige Ergebnisse wurden bxi der Gärung erzielt, als bei Würzen aus sehr carbonatreichem Wasser die Würzeacidität durch Zusntz von Mine.

44) Biochem. Z. 36, 477.

45) J. biolog. (hem. 10, 169.

46) Wochenschr. f. Brauerei 28, 61 .

47) Ibid. 17, 713, 762.

48) Ibid. 28, 393; diese Z. 24, 2131 (1911) (1911).

49) \%. f. Spiritus-Ind. 34, 86; diese Z. 24, 1548

so) Jahrb. Vers. u. Ieliranst. f. Brauerei 14, 43. ralsäure etwas erhöht wurde, jedoch nur so weit, daB die Würze gegen Methylorange noch alkalisch rengierte. Benutzt wurden Phosphorsäure, Schwofol. süure und Salzsäure. G. F e ue rate in ${ }^{51}$ ) hat Säurewaschung mit günstigem Erfolg zur biologischen Reinigung infizierter Hefe benutzt. Nach

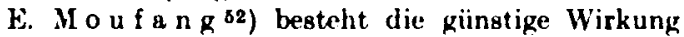
bei Betzandlung der Hefe nit verd. Phosphorsüure darin, daB cimmal Verunreinigungen gelöst, infizierende Organismen beseitigt werden, und die Hofe angeregt wircl. Süureempfindliche Hefen lassen sich nit gutem Frfolg durch Wanchen mit Phosphatlösungen behandeln.

Bei der biologischen Unterauchung von li ra u w äs sern ist nach $H$. W i ll ${ }^{53}$ ) für die Beurteilung die Zahl der Organismen ohne wesentliche Bedeutung. Als wichtigste: Prüfung ist dic Widerstandsfähigkeit der Wasserorganismen gegen gärende $\mathrm{H}$ (fe durch die Gürprole zu ermitteln. Vf. gibt ein Verfahren zur Anstellung dieser Probe an.

II. W. B e ijerin c ${ }^{54}$ ) veröffentlicht Boobachtungen über die l'igment bild ung bei Ess a i b a k terien. In leichten Biersorten entwickelte sich spontan ein vom Vf. als Acetobacter melanogenum inzoichnetes Essigbakterium, das durch die Fähigkeit, einen tief braunen Farbstoff zu bilden, nusgezeichnet war. Der Farbstoff ist ein aromatischer Stoff, vielleicht identisch mit Chinon. J. $\mathrm{H}$ o $1 \mathrm{~m}$ sb) gibt eine Methode zum $\mathrm{Nachweis}$ von Mykoderma in Brennereien und Preßhefefabriken $\mathrm{an}$.

Von Arbeiten über $H$ of fonenzy mo betreffen einige die $H$ efe $\mathrm{n}$ i n vert a se. H. Euler und S. K u ll bor $g^{56}$ ) haben versucht, die Invertase aus autolysierter Hofe rein darzustellen. Die Eiweibstuffe des Hefensnftes wurden mit Bleiacetat und und Kaolin entfernt, die Aninosäuren durch IDialyse. Aus der Diffusionageschwindigkeit berechnen Vff. für die Invertase ein Molekulargewicht von ungefähr 27000 . Dic lösungen des Enzyms zeigen eine ihrem Invertasegehalt proportionule Wirksamkeit. Von A. P. II a $t$ he w a und T. H. G l e n n ${ }^{57}$ ) aus Hofe gewonnene Invertnse enthiclt ca. $1 \%$ Asche und $2,20 \%$ stickstoff. Jurch Säurehydrolyse lassen sich etwa $70 \%$ des knzyms als reduzierender Zucker abspulten, der Zucker erwies sich als in der Hauptsache aus Mannose bestehend. Vff. betrachten nach diesen Hefunden die Invertase als eine Verbindung cines kiweiBstoffes mit einem Mannosan. In Fortsetzung ihrer früheren Arbeiten ${ }^{58}$ ) ïber chemische Zusammenset zung und Bildung von Enzymen beobachten $H$. E $u$ le $r$ und $B$ e $t h$ a $f$ Ug $\left.\operatorname{lag}^{58}\right)$, daB Behandeln der Hefe mit Natriumphosphat den Invertusegehalt erhöht, clenso

s1) Wochenschr. f. Brauerei 28, 16; diese Z. 24, 1051 (1911)

52) Ibid. 28. 410.

5a) Z. ges. Brauwesen 34. 125; diese $Z$. 24, 1048 (1911)

56) Zentralbl. Bakt. u. Parnsitenk. II, 29, 169.

55) Bll. de l'Assoc. des ('him. de Nuer. et Distill.

28, 1040

s8) Z. physiolog. (hem. 73, 335.

67) $Z$. biolog. (hem. 9, 29 .

58) Diese Z. 24, 778 (1911).

59) Z. physiolog. (hem. 70, 279. 
wie den Zymasegehalt. DaB die Hefedauerpräparate soviel schwächere Gärwirkung entfalten als die lebende Hefe, liegt nach der Vff. Ansicht daran, daB die Zymase in der lebenden Zelle zum größten Teil an Protoplasma gebunden ist. Bei der $\mathbf{A b}$. tötung des Plasmas wird der gebundene Enzymanteil inaktiviert, aktiv bleibt nur der vom Plasnia abgespaltene Zymaseanteil. In einer weite. ren Arbeit zeigen $H$. Eu ler und $S$. $K$ ull ber $\mathbf{g}^{60}$ ), $\mathrm{da} B$ die Invertasewirkung durch lebende Hefe eine monomolekulare Reaktionskonstante aufweist. Fs ist anzunehmen, daB die Invertase nicht wie die $Z y$. mase zum yrößten Toil an Plasma gebunden ist, der Temperaturkoefficient ist bei lebender Hefe der gleiche wie bei Invertasepräparaten. Ias Verhältnis Inversionskonstante zur Gärungskonstante weist, da die Inversion stets viel schneller verläuft als die Gärung, sehr hohe Werte auf; diese Quotienten scheinen eine für die verschiedenen Heferassen charnkteristische Gröbe zu sein. Dieselben V'ff. ${ }^{61)}$ erklär('n (las virschiedenartige Verhalten der Hefenenzyme, Zymase, Invertase, Multase, hinsichtlich ihrer Fxtrahierbarkeit aus lebender und aus trockener Hefo und hinsichtlich ihrer Widerstandsfähigkeit gegen Antiseptica durch die bereits angedeutete Annahmr. daB diese Fnzyme entweder an l'rotoplasma gebunden sein können oder von demsalben als unabhängige Stoffe abgeschieden werden. Im letzteren Falle sind sie leicht extrahierbar und gegen Antiseptika riemlich widerstandsfähig, im letzteren Falle lassen sie sich nur schwierig aus der lebenden Zelle extrahieren und sind dann gegen Antiseptical schr empfindlich.

A. v. Le bedeff $\left.\left.{ }^{62}\right)^{63}\right)^{64}$ ) beschreibt in einer Reihe von Arbeiten eine schr einfache Methode zur (lewinnung gärkräftigen $H$ efesaftes. Abgepreßte Hefe wirl in dünner Schicht bei 25 bis $30{ }^{\circ}$ höchstens 2 Tage lang getrocknet. Diese Trokk:nhefe gibt beim Incerieren mit der zweieinhalbbis dreifachen Menge Wasser einen Macerationsr.lft, dor starke (Härkraft zeigt. Dic getrocknete Hefe ist auzgezeichnet haltbar, so dab sich nach monatelangem Auflowahren noch gärkräftige Säfte

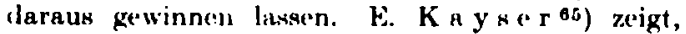
daB die Wirkanmeit des $L$ e b e d e f f schen Saftes in hohem Maßs. vom Zustande der Hefe, von der Art des Trocknons und vor allem von der Macera. tionstemperatur abhängt. Ein Zusitz von (ilycerin zum Macerationswasser ergibt haltbarerc, aber we. niger wirksame Säfte. Kine weitere Methode zur Gewinnung aktiver Hefensäfte gibt $\mathbf{P}$. R in ck le be ${ }^{66}$ ) an. Er plasmolysiert Hefe mittels Gly. cerin. In dem dabei erhaltenen Safte wird das Koenzym leicht zerstört. ist dics der Fall, so gelingt die Aktivierung des Safter lejcht durch Zusatz von Kochsaft.

In ciner Arbeit ïber den $\mathrm{E}$ influB der Antiseptica a ufdie Hefenautolyse

60) Ibid. \%1, 14.

61) Ibid. 73, 85 .

62) Compt. r. d. Acsd. d. Bciences 152, 49.

6) Bll. Soc. Chim. de France 9, 744; diese Z. 25, $185(1912)$.

64) Z. physiolog. Chem. 73, 447.

os) Compt. r. d. Acad. d. sciences 15\%, 975.

60) Chem.-Ztg. 35, 1149. stellt E. Navasaart ${ }^{67}$ ) fest, daß eine befördernde Wirkung bestimmter Antisepticakonzentrationen auf die proteolytischen Hefenenzyme nicht zu brobachten ist. Nur die Nuklease wird durch Sinfölwasser etwas angeregt. Formaldehyd in $1^{\circ}$ iger Lösung hebt die Autolyse auf.

Gegenstand eines Vortrages von F. Fcrnba c h ${ }^{68}$ ) ist die Rolle der l hos phorsäure in der Brauerei. Die anorganischen Jhosphate, die sich bei der Keinung aus den organiachen Phosphorsäureverbindungen der Gerste gebildet haben, sind von großem Hinfluß auf den Verlauf der enzymatischen Vorgänge bei Maischen, sie spielen weiter eine wichtige Rolle bei der Ernährung der Hefo und als Regulator der Gärung. I le noch im Bier verbleibenden Phosphatmengen Ixeinflussen die Haltbarbeit günstig.

H. Euler und A. Fod or ${ }^{69}$ ) haleen an Hefegum mi, der nach $S a l k o w s k$ is . Methode gewonnen war, einige lintersuchungen angestellt. Das Molekulargewicht liegt ïber $1000 \mathrm{~m}$. Bei der Hydrolyse: wurden nur Mannose und (ilucose erhalten, keine Pentosen.

Sehr zahlreich sind wiederum die Arbeiten, die sich mit dem Verlauf, dem Chamismus und der l) yn a mik der Gärung befasson. Zunächst zeigen einige Arbeiten, daB die Hefe imstande ist, eine große Zahl anderer Stoffe als Zucker zu vergären, d. h. in einfachere Stoffe zu zerlegen. So haben H. F r a $n z$ ( $n$ und $\left(0\right.$. S t e p ph u ${ }^{70}$ ) beobachtet, daß $N$ a t r i m for $m$ i a t durch eine ganze Reihe von $H$ efen vargore $n$ wird. In Ameisensüure auch gebildet wird, ohne da B Aminosäuren als Stammsubstanz in Frage kommen kön. nen, neigen Vff. zur Ansicht, dnB Ameisensäure eine Rolle als Zwischenprodukt der Alkoholgärung spielt. (. N e u be r $\left.\mathrm{g}^{71}\right)^{72}$ ) zeigt in einer Anzshl Arbeiten (gemeinsam mit $\mathbf{l}$. $K$ a r c z a g und $\mathrm{L}$. $T$ i $r$ ), daß außer Ameisensäure auch Essigsäure, Buttersäure, Oxysäuren, Oxalsäure und Homologe, vor allem aber a-Ketosäure, wie besonders die Brenztraubensäure Lxi Abwesenheit von Zucker durch Hefe, auch durch l)auerpräparate, in Form ihrer Calciumsalze unter Kohlendioxydabspaltung vergoren werden. Vf. schreibt diese Wirkung ein'm Enzym, der Ca r bo x y la s e zu. Die Vergärunt der Brenztraubensüure vollzicht sich nach der Formel:

$$
\mathrm{CH}_{3} . \mathrm{CO} . \mathrm{CO}_{2} \mathrm{H}=\mathrm{CO}_{2}+\mathrm{CH}_{3} \cdot \mathrm{CHO} \text {. }
$$

Es ist von hohem Interesse, daß nach Beobachtungen von $H$. Euler ${ }^{73}$ ) ultraviolettes L i c h t ähnlich zersetzend wirkt und $z$. B. Milchsäure, viel stärker aber Brenztraubensäure unter Kohlendioxydabspaltung zersetzt. Beiläufig mag hier auf eine Mitteilung $L$. M a 8 sols 74 ) hingewiesen sein, nach der ultraviolet tes L i cht auoh verzuckernde Wirkungen

67) Z. physiolog. Chem. 72, 151. (1911).

88) Z. ges. Brauwesen 34, 359; diese Z. 24, 1985

6) Z. physiolog. Chemie 72, 339.

70) Ber. 44, 2915.

71) Biochem. Z. 36, 60.

72) Ibid. 37, 170.

73) Z. physiolog. Chem. I1, 311.

74) Compt. r. d. Acad. d. sciences. 152, 902. 
in Stärkelösungen reduzie rende Zucker, wahrscheinlich Maltose, und etwas durch Alkohol fällbare Subetenzen (Dextrin?) bil det. Auch durch Radioaktivität sollen die Gärer. scheinungen beeinfluBt werden. W. C a s p a ri ${ }^{25}$ ) hat sich ein Brauverfahren unter An. wendung von Rndiogktivität patentieren lassen, bej dem durch Emanation Wachstum und Tätigkeit der Hefe, besonders hinsichtlich der Bildung von Bukettstoffen günstig beeinflußt werden soll.

Hinsichtlich der Ansichten über die $\mathrm{R}$ olle der Phosphorsäurebei dereigent. lichenalkoholischan Gärung ist eine gewimae Klärung eingetroten. A. v. Lobe . de $\left.\left.w^{76}\right)^{75}\right)^{r y}$ findet jetzt dic Angalen von $H$ a $r$.

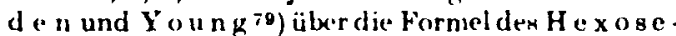
phosphorsäureesters zu $\mathrm{C}_{6} \mathrm{H}_{10} \mathrm{O}_{4}\left(\mathrm{H}_{2} \mathrm{H}^{2} \mathrm{O}_{4}\right)_{2}$ bestätigt. In Vf. den gleichen Ester bei der Vergärung von Dioxyaceton durch Hefemacerationswaft beobachtet, iat er der Ansicht, dal als erste Phawe der Alkoholgewinnung ein Zerfall der Hexose in 2 Molekiile Triose zu betrachten ist, und daB sich der Gärungsverlauf durch nachstehendes formel. schenta wiedergeben läBt:

$$
\begin{aligned}
& \text { I. } \mathrm{C}_{6} \mathrm{H}_{12} \mathrm{O}_{6}=2 \mathrm{C}_{3} \mathrm{H}_{6} \mathrm{O}_{3} \text {, } \\
& \text { II. } 2 \mathrm{C}_{3} \mathrm{H}_{6} \mathrm{O}_{3}-2 \mathrm{RHP} \mathrm{O}_{4}=2 \mathrm{C}_{3} \mathrm{H}_{5} \mathrm{O}_{2} \mathrm{RPO} \text {, } \\
& 2 \mathrm{H}_{2} \mathrm{O} \text {, } \\
& \text { III. } 2 \mathrm{C}_{3} \mathrm{H}_{5} \mathrm{O}_{2} \mathrm{RPO}_{4}=\mathrm{C}_{6} \mathrm{H}_{10} \mathrm{O}_{4}\left(\mathrm{Rl}^{\prime} \mathrm{O}_{4}\right)_{2} \text {, } \\
& \text { IV. }\left({ }_{6} \mathrm{H}_{10} \mathrm{O}_{4}\left(\mathrm{RPO}_{4}\right)_{2}+\mathrm{H}_{2} \mathrm{O}=\mathrm{C}_{2} \mathrm{H}_{6} \mathrm{OH}\right. \\
& +\mathrm{CO}_{2}+\mathrm{C}_{3} \mathrm{H}_{5} \mathrm{O}_{2} \mathrm{RPO}_{4}+\mathrm{HRPO}_{4} \\
& \text { oder } \\
& \text { V. } \mathrm{C}_{6} \mathrm{H}_{10} \mathrm{O}_{4}\left(\mathrm{RPO}_{4}\right)_{2}+2 \mathrm{H}_{2} \mathrm{O}=2 \mathrm{C}_{2} \mathrm{H}_{5} \cdot \mathrm{OH} \\
& +2 \mathrm{CO}_{2}+2 \mathrm{HRPO}_{4} \text {. }
\end{aligned}
$$

Auch $\mathrm{H}$ ardon und $Y(1) \mathrm{ng}^{80}$ ) bestätigen, im (iegensatz zu den früheren Angaben Le bed eff $\mathrm{g}^{81}$ ) bei erneuten Fersuchen dic Richtigkeit der oben erwähnten Formel des Hexosephosphorsäureesters bestätigt. Sie beschreiben die Eigenschaften dieser Verbindung und einer Anzahl Derivate derselben. Die nähere Formel des Eaters iat wahrscheinlich $\mathrm{H}_{2} \mathrm{I}^{\prime} \mathrm{O}_{4} \cdot \mathrm{C}_{4} \mathrm{H}_{8} \mathrm{O}_{3} \cdot \mathrm{C}^{\prime} \mathrm{O} \cdot \mathrm{CH}_{2} \cdot \mathrm{H}_{2} \mathrm{POO}_{4}$. Aulser diesem Hexosediphosphat scheint nach Beobachtungen von H. Euler und A. Fod or ${ }^{82}$ ) noch ein Monophos phat zu entstehen. Die naheliegende Annahme, daß die gärungsbeschleunigende IV irk ung von Arseniaten bei PreBaftgärungen in glcicher Weise zustande komme wie die der Phosphate, kann nach Versuchen von $A$. $H$ a rden und $W$. Y o un ${ }^{83}$ ) nicht zutreffen, das Arseniat geht dabei nicht in organische Bindung uber. IBis zum Erreichen einer Optimalkonzentration beschleunigt Arseniat den Zerfall von Glucose, Mannose, fructuse. In höherer Konzentration wirkt Arseniat hemmend. Auch die Selbstgärung des

76) D. R. P. 235 173; diese Z. 24, 1455 (1911)

76) Compt. r. d. Acad. d. sciences. 133, 136

77) Biochem. Z. 36, 248.

78) Ber. 44. 2932.

79) Diese Z. 24, 779 (1911).

80) Biochem. Z. 32. 173.

81 ) Ibid. 28, 213.

82) Ibid. 36, 401 .

83) Proceed. Royal Suc. Londun B, 83, 45l.
Preßeaftes und die Glykogengärung wird beschleunigt. Arsenite wirken ähnlich, aber schwächer.

Aus einer Arbeit: Beitrag zur $K$ en $n$ t $n$ is der Dynamik der Hefegärung von $H$. E u l er und G. L und q u i $\mathrm{t}_{\text { }}$ ) ) mögen folgende Beobachtungen erwähnt sein. In der ergten Hälfte ist bei Maltosegärung die Menge der entwickelten Kohlensäure der Zeit proportional. Der Zerfall von Maltose ist fast ebenso schnell wie der von Glucose. Die Hydrolyse der Maltose scheint nicht wesentlich schneller zu verlaufen als die Gärung. Auf die Mannosegärung, die langsamer als die Glucose. gärung verläuft, ist Mononatriumphosphat olıne EinfluB. R. O. Herzog und $O$. Salad in ${ }^{A 5}$ ) haben die Anderung des färvermögena bej Uberführung lebender Hofe in A cetond a uerhefe zum Gegenstand einer Arbeit gemacht. Lebende Hefe und Dauerhefe, die gegenüber Glucose die gleiche (iärkraft besitzen, zeigen anderen Hexusen gegenüber völlig anderes Verhalten. Wenn die lelxende Hefe am raschesten bei Glucose, langsamer bei Fructose und am lang. samsten bei Mannose wirkte, yo vergärt die Acetonhefe die Fructose am schnellsten und Mannnse am wenigsten schncll. Die (ieschwindigkeitskonstante berechnet sich am lesten nach der Formel:

$$
v={ }_{t}^{1} \log \frac{C_{0}+\left(C_{0}+C_{t}\right)}{C_{t}}
$$

A. S I a tor ${ }^{86}$ ) zeigt in einer: $\mathrm{C}^{\dagger} \mathrm{b}$ e $\mathrm{r} d \mathrm{o}_{\mathrm{n}} \mathrm{Gr}$ a d der alkobolischen Gärung betitelten Arbeit, daß der Vergärungsgrad, gemessen durch den Druck der entwickolten Kohlensëure, bis in 150 Mill. Zellen in 1 cen (?) proportional der vor. handenen Hefenmenge ist. Die Zuckerkonzentration ist bei mittleren Buträgen ohne wesentlichen FinfluB auf dic: Gärung. Glucose, Fructose, Saccharose und Maltuse lassen keinen Unterschied im Gärungsgrad erkennen. Der Temperaturkoxeffizient ist sehr groß, er beträgt für Glucose boi $20^{\circ}$ gegenüber einer Temperatur von $10^{\circ} 3,6$.

Wie F. Ehrlich si) findet, wird auch das $\mathrm{T} y \mathrm{rosin}$ in analoger Weise wie die anderen Aminosäuren vergoren, und zwar liefert cs dabei p. Oxyphen $y$ läth ylalkohol, den Vf. als $\mathrm{T}$ y $\mathrm{r}$ os o I bezcichnet, und der ein ganz allge. mein vorkommendes Gärungsnebenprodukt zu sein scheint. Nebenher entsteht noch etwas p-Oxyphe. nylmilchsäure oder p-Oxyphenylessigsäure. Derselbe Vf. hat gemeinsam mit K. A. J a cob s e ${ }^{\text {s8) }}$ das Verhaiten von Schimmelpilzen gegen über von Aminos äuren studiert, die sich meist ganz anders verhalten als Hefe. Bei Abwesenheit von Kohlehydraten findet meist ein sehr weitgehender Abbru der Aminosäuren statt, in anderen Fällen sind die Umwandlungen keine: tiefergreifenden. So wandelt z. B. Oidium lactis, Monilia candida und eine Anzahl Mukorineen die natürlich vorkommenden Aminosäuren unter Ammoniakabspaltung in die entoprechenden Oxysäuren um. Die Reaktion verläuft so glatt, daß sie zur

84) Z. physiolog. Chem. 78, 97.

85) Ibid. 73, 263.

88) J. Inst. Brewing 17, 147; Wochenschrift f. Brauerei 28,141 .

87) Ber. 44, 139; diese Z. 24, 1050 (1911).

88) Ibid. 44, 888 . 
Darstellung solcher Oxysäuren bənutzt werden kann. O. N e u b a u e $r$ und $K$. F r o $m$ he r $z^{89}$ ) konnten zeigen, $\operatorname{daB}$ bei $\operatorname{dem} A \mathrm{~m}$ ino $\mathrm{x} \ddot{\mathrm{u}} \mathrm{re}$ - a b b a u d u r c h H e f e, gerade so wie im Säugetierorganismus, intermediär Ketosäuren entstehen. Als Schema für den Abbau geben sie das folgende:

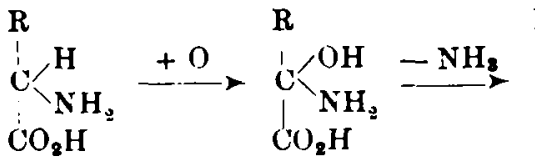

Als Nebinprodukt entstehen Alkoholsüuren $\mathrm{R}-\mathrm{CH} . \mathrm{OH}-\mathrm{CO}_{2} \mathrm{H}$. Furfurol wird nach C. J. L, i n t ne r und $H$. J. v. L i e b i ${ }^{{ }^{\circ 0}}$ ) durch gärende Hefe zu Furfurylalkohol reduziert. Auch nichtgärende, im Wasser nufgeschlämmte Hefe zeigt Rexluktionswirkung, aber schwächer. Als Nebenprodukt entsteht ain beti $50,5^{\circ}$ schmelzender, dem Furfurylalkohol ähnlich riechender krystalliner Stuff. I, M a th $\mathrm{i} \times \mathrm{n}^{\theta 1}$ ) hat gelegentlich dio Bildung von Xthylmercaptan bai der alkoholischen Gärung beobachtet.

In einer Arbeit: $H$ op fe n u nd Hefo zcigt H. W. H a r m a $n{ }^{9: 2}$ ), da 3 die spezifische Hopfenwirkung nur beim Hopfenkochen zutuge tritt. Boi allen anderen Verfahren des Hopfens erzielt man meist schlecht haltbares Bier ohne Verbesserung des Hopfengeschmacks. Alozuraten ist von der Verwendung von Hupfenextrakten. Der Stickstoffgehalt der mit Hopfen gekochten Würzen ist nur unbedeutend geringer als der Würzen, die nicht mit Hopfen gekocht wurden. Auf die Hefotätigkeit wirkt der Hopfen anregend. Wie Versuche an Zucker-Peptonlösungen zeigten, ist dio von der Hefo h:rausgenommene Stickstoffmenge unabhängig vom Stickstoffyehalt der Würze. Auch dierer Vf. bestätigt. daB die Hefenernte von der Aussaat unabhängig ist. Dem Verein d e $\mathrm{r} S \mathrm{p}$. ritusfabrikantenin Doutse hland ${ }^{\text {93 }}$ ) ist ein Verfahren zur Vorgärung von Brennereimaisehon unter Verwendung von Brauereih ef e patentiert worden. Fin Teil der Hefe wird nit vermaischt, cin anderer wird zum Anstellen der (iïrung benutzt. Fin Gärungs -

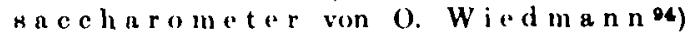
bestimmt den Druck der bei der (jürung entwikkelten Kohlensäure manometrisch. Als Manometerflüssigkeit dient die (Gürflüssigkeit selbst. I. v o n Vet $t$ e $^{95}$ ) macht Angaben über die Verwen dung von ozon im Braucrabetrieb. Dis Ozon kann benutzt werden zur desinfizierenden Behandlung der Roh- und Betriebsstoffe, von (Yeräten und als Zusatz zur Frischluft bei der (yärkellerventilation. Finwirkung von luft mit $3 \mathrm{~g}$ Ozon auf $1 \mathrm{cbm}$ st:rilisiert die Hefe, höhere Ozonkonzentrationen tïten auch die Hefe. Zum gleichen Thema siehe auch tine Veröffentlichung von $I$. v. Vet ter und li. Mouf $n \mathrm{n} \mathrm{g}^{96}$ ).

s9) Z. physiolog. ( hem. ro, 326.

80) Ibid. 72, 449.

91) Bll. de l'Awsoc. des Chim. de Sucr. et Distill. 28, 971

92) Wochenschr. f. Brauerei 28, 385; diese Z.

24, 2130 (1911).

93) D. R. P. 236486.

94) D. R. P. 232009 ; diese Z. 24, 605 (1911).

๑5) Wochenschr. f. Brauerei 28, 13; diese Z. 24,

1049 (1911).

96) Ibid. 28, 377 .
$\mathbf{R}$

co

$\mathrm{CO}_{2} \mathrm{H}$

C'ber die weiteren Fortsohrit te auf dem Gebieteder Hefoverwertung berichtet F. $\mathrm{H}$ a $\mathrm{y}\left(\mathrm{l} \mathrm{u} \mathrm{c} \mathrm{k}^{97}\right)$. Als wichtigste Verwertungsart scheint sich dicjenige der entbitterten und getrockneten Hefe als Nährpräparat zu entwickeln, die bislang gesammelten praktiachen Erfahrungen sind durchweg günstig. Wxakte Versuche von W. $V$ öltz und $A$. $B$ a $u$ d $r\left(x \in 1^{\circ 8}\right)$ erganzen die von diesen Vff. früher gemachten Beobachtungen ${ }^{90}$ ). Auch im m:nachlichen Organismus ist die Ausnutzung der Trockenhefe eine sehr gute. I ie mittels Soda entbitterte Hofo zeigte folgende Zusammengetzung: $6,87^{\circ}{ }_{\circ}$ Wasser, $7,04^{\circ} \%$ Asche, 50,04\%, Reinciweik, 3.12\% Fett, 1,44\% Rohfaser und $28,09 \%$ N-freje Extraktstoffe. O. v. C z a d a $k^{100}$ ) hat Fitterungaversuche mit Trockenleefo an Sehweinen angestellt, die gleichfalls za sehr günstigen Frgebnissen fïhrten. (). F a 11 a d a 101) hat aus $\mathrm{g}(0-$ trockneter Hefe und Molasse cin halt barex und wert volles futtermittel hergestellt.

\section{V. (; ärunguerze ugnisse.}

In einer längeren Arbeit: Ursachen des A usacheidens von Fiwa i $B$ a u for tigem fila $\mathrm{ach}$ en bier trotz normaler B $^{2}$. handlung im Sudhaus und Keller erörtert F. Fi m 8 l a n d e $r^{102}$ ) vom kolloidehemischen Standpunkte aus die chemischen und physikulischen Lrsachen, die auf der cinen Site eine Stabilisierung des kolloid gelösten Fiweibes bewirken, und dic andererseits diese Lïsungen zur Ausflockung bringen. Stabilisierend wirken in erster Linie die im Biere vorhandenen sauer reagierenden Stoffe, sofern nicht eine optimale Wasserstuffionenkonzentration iiberschritten wird. Auch dic Hopfenharze wirken in der gleichen Richtung. IDe Ausflockung begünstigen starke mechanische Erschütterungen, zu hoher Kohlensäuregehalt usw. Eine andere kolloid. chemische Arbeit von A. R o i c h a r d 103) handelt über $\mathrm{M}$ alz- und $\mathrm{H}$ opfengerbstoff. Der erstere findet sich zunächst in gröber dispersem $\mathrm{Zu}$ stande in den Malzauszügen und geht erst alhmählich in einen feineren I)ispersionsgrad über. Der Hopfengerbstoff scheint aus zwei Komponenten zu bestelien, die infolge verschiedenen Dispersitätsgrades verschiedenen fällungbwert gegenüber Ei-

97) Jahrb. Vers. u. Iehranst. f. Brauerei 14, 282

98) Wochensehr. f. Brauerei 28,85 ; diese $Z$. 24 , 1097 (1911).

99) Diese' Z. 24, $779(1911)$. 214.

100) J. landwirtach. Versuchswesen Osterr. 14,

101) Osterr.-ungar. Z. Zuckerind. 40, 709.

102) Kolloidehem. Heiliefte 3, 47; diese 7.23 , $186(1912)$.

103) Z. ges. Brauwesen 34, 253; diese Z. 24 . $1986(1+11)$. 
weiB besitzen. Nach 1 . $R \circ h$ la n d 104) wirken von den Bierkolloiden, welche für die $\mathrm{S} \mathrm{ch} \mathrm{a} \mathrm{u} \mathrm{m}$. bild Ing des Bieres in Betracht kommen, besonders günstig die Hopfenharze. Fine andere wichtige Rolle der Kolloide ist nuch lf. Ansicht auch ihre Adsorptionswirkung gegenüber der Kohlensäure, sus dor sich die Kohlensiäurehaltung def Bieres erkläre. Dicse Ansicht scheint nach Versuchen von $A$. Find $\mathrm{A} y$ und $B$. S h $\mathrm{n}^{105}$ ) nicht zutreffend zu wein, nucl deren Versuchen ergab sich, daB die las lichkeitronkohlensä tre

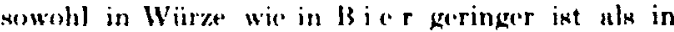
kolloilfreien Alkohol-Wassermischungen. Bei Bier sinkt die Lïslichkeit mit ste-igendem Alkoholgrehalt. Dic diesen befunden widersprechenden älteren An-

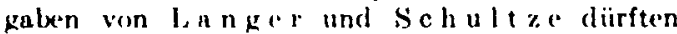
sich aus den C'mstande erklären, daß diese dutoren

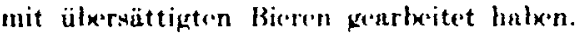

Nach F. II o uf a n $\mathrm{g}^{108}$ ), der eine Arbeit: Kältermptindlichkeit und $\mathrm{Halt}$ bar. keit dor Biere veröffentlicht, sind die Eiweiß. stoffe, welche AnlaB zu FiweiBtrübungen geben können, im Biere teils an Süuren, teils an Baren und teils an Sul\% gebunden. (iünstig berinfluBt wird Haltbarkeit und Kältuempfindlichkeit nach Vf.'s Ansicht durch genügenden Sëuregehalt, besonders aber durch einen Gehalt des Brauwaswers un Mag-

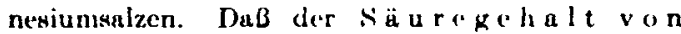
grober Bedeutung für die Haltbar. $k$ e it des Bieres ist, zeigen Versuche ron $F$. sehoenfeld und W. H i r t 107). Zumatz von Milchsäure zum fertigen Biere erhält diess's länger biank. Wird aber einc Optimalkonzentration ïberschritten, wo triibt sich das gexätuerte Bier sehnoller als das ungesäuerte, dic Ausscheidungen bestehen nus Hopfenharz. Auf Alkulizusatz erfolgt rasche Fituicklung von Mikroorganismen, namentlich inarzina.

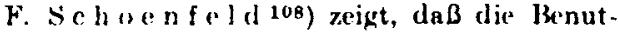
zung der Ballingsehen Formel zur Be. rechnungdesstanmw iurzegehaltes zu hohe Werte ergibt wegen des zu hohen Hefevermehrungsfaktors von $B$ a I I ing. Bei obergärigen Bieren werden wegen ointretender Alkoholverdunstung und stärkerer Hefenvermohrung etwak. zu niedrige Werte erhalton. If. empfichlt, an stelle der $B$ a $l$ ling schen Formel dio nachstehende zu benutiten :

$$
p=\left(\frac{2,0195>A+n}{1,0195 ;}\right) \times 100
$$

Modifizicrte Formeln zur Stamumwïrzenberechnung gelen auch I) o e me $n$ s und F. P'a w low a k i ${ }^{100}$ ).

A. B a u ${ }^{110}$ ) beschreibt eine Methode zur A 1 . kuholbestimmung im Bitre wecks rascher Feststellung, ob das Bier ïber oder unter 1,75 MnBprozent Alkohol enthält. Diese Methode

104) Wochenschr. f. Brauerei 2R, 273; diese $Z$. 24. 1987 (1911).

106) J. (hem. Sor. 99, 1313; diese Z. 25, 187 (1912).

106) Wochenschr. f. Brauerei 28, 501 .

107) Ibid. 28. 467; diese Z. 25, 187 (1912).

108) Ibid. 28, 209; diese Z. 24, 1547 (1911).

109) Z. gex. Brauwesen 34. 369; diese Z. 24, $2131(1911)$

110) Wochenschr. f. Braucrei zx. 55. hat groBe praktiacho Bedeutung, weil in der kommunalen Bierbesteuerung die Biere mit weniger als $1,75^{\circ}$, Alkohol nur mit einem geringeren Steversatz belegt werden dürfen. E. Poz zi $-E \mathrm{scot}$ 111) gibt eine Methode zur Beatinmung de-s De $x$ trins i m Bier un. Das eingeencte Bier wird mit Alkohol gefällt, zentrifugiert, der Nieder. schlag wieder gelöst, abermals gefällt, nit Ather oder Accton gewaschen und dann bei $80-90^{\circ}$ ïber Schwefelwäire petrocknct. Zur B est im m un kloiner Hengenven Bleiim Biereignet sich eine von $\mathrm{A}$. W. $\mathrm{K} \mathrm{n}$ a $\mathrm{p}^{112}$ ) angegelene colo. rimetrische Methode.

K. ․ 13 a $\left(\mathrm{h} \mathrm{k} \mathrm{a}^{213)}\right.$ berichtet aber Beobachtungen letreffend die Alkoholktärke von Trinkbranntweinen. Der ullgemein zu beobuchtende Ribkgang in der Alkoholstärke wockt den Virdacht, daB lx.hufw Vordecken der geringen Stärke Branntweinschärfen Verwendung finden. Vf. regt die Verstündigung der beteiligten Kroise über fo'stzulegendo Minde'stgrenzen des $\mathrm{Al}$ koholgehaltes fiir Trinkbranntweine an.

Die von $\mathrm{Holl}$ aende $\mathrm{r}^{114}$ ) angegelene Methode zum $\mathrm{N}$ ach weis von $A$ my lalkohol in Branntwein ist nach F. Herzog 115) nicht brauchlur, da die der Methode zugrunde lie. gende Reaktion eine Furfurolreaktion, keine Amylalkoholreaktion ist. Die höheren Alkohole i m . I $m$ a ic a $r$ um bestehen nach Th. van Fellenbergie) in der Hauptsache aus nButylnlkohol, dem viellejeht sehr geringe Mengen [ropylalkohol leigemengt sind. Isobutylalkohol und Amylalkohol sind höchstens spurenweise vorhanden.

('. Ordonnen $\|^{117}$ ) gibt eine Formel zur Best im mung des Alkoholgeh al"tas zuk k c rhaltiger Lik ïr aus dem scheinbaren Alkoholgehalt und dem Extraktgehalt. Zum Nach weis kloingr Alkoholmen'gen in gürenden Flüssigkeitun usw. kann nach $\mathrm{A}$. K lö ck $\mathrm{er} 118$ ) folgende Methode dienen: Kinige Kubikz'ntimeter der betreffenden Flïssigkeit werden in einem Roagensplas mit aufueset\%(em Steigrohr als Kiiller zum Sieden erhityt. Bei (iegenwart auch kleinster Mengen von Alkohol beobachtet man am Kühler die Bildung , ̈̈liger" Tropfen Kondensates. F. J n n a ${ }^{11 \theta}$ ) beschreibt einen $A$ p a $r a t \% u m$ dlkoholnachweis in Lut ter. by wirl dic Reduktion von Chromsäure durch Alkohol zum Sachweis benutzt.

Die (hemische Fubrik (irieshe-im-Elektron 120)

11) Bll. de l'Asroc. des (him, de ${ }^{-}$Sucr. et Dixtill. 29. 72.

112) J. Suc. (hem. Ind. 30, 165; diese Z. 23, 187 $(1912)$

113) J. Unters. Nahr.- u. GenuBm. 22. 34

114) Diese Z. 24, 78I (1911).

115) Z. Unters. Nahr.- u. GenuBm. 21, 280 ; diese Z. 24, 854 (1911). $3 \overline{2} 2$.

16) Mitt. Iobensmittelunters. u. Hygiene 1,

117) Ann. Chim. analyt. 16, 139; diesc Z. 24, 2075 (1911).

118) Zentralbl. Bakter. It. Parasitenkunde II, 31, 108

119) Osterr. Chem.-Ztg. 14, 17

120 ) D. R. P. 236591 . 
stellt a b s o l u te $\mathrm{n}$ A 1 k o h o 1 her durch Behandeln von Spiritus mit mehr als $80 \%$ Alkohol mittels entwasserten Schwefelnatriums. J. T r \& u b e ${ }^{181}$ ) ist ein Verfahrenzur Reinigung von Rohspiritus durch Schichtenbil. d u n g psitentiert worden. Dem Rohspiritus wurden Chlornatrium- oder Chlorkaliumlösungen unter $\mathrm{Zu}$ satz von Salzen mit zwei- und mehrbasischen Säuren und Basen zugesetzt. J. Klug e ${ }^{122}$ ) will Branntw e i n v e r g äl l e n mit den von Wasser befreiten, bis $180^{\circ}$ übergehenden Destillationsprodukten aus Steinkohlenteer.

Der Mas chinenbau-A.G. Golzern. Grimma und A. Zeckendorf ${ }^{123}$ ) ist ein Verfahren patentiert worden zur Gewinnung des bei dem Durchlüften gärender Maischen, insbesondere bei der Lufthefefabrikation, mit der Luft entweichenden Alkohols. I ie alkoholhaltige Luft wird vor dem Einleiten in die zum Niederschlagen des Alkohols dienende Vorrichtung mit Wasserstaub, Wisserdampf oder anderen Alkohol absorbierenden Dämpfen vernischt.

Zur A usbeutabestimmunginschnell. essigfabriken können nach $H$. Wüsten feld 124) zwei Berechnungsarten in Frage kommen. entweder aus regclmäBigen Analysen von leichter Maische und Ablaufessig und Feststellung der verbrauchten Maischemenge und des gewonnenen Essigs oder indirekt durch Firmittlung der zu Anfang und Schluß eines bestimmten Zeitnbschnitts vorhandenen Menge Denaturnt, lejehter Maische und Fissig.

F. Rothenbach und H. Wüsten fe (d 125) schildern die innere Beachaffen. hoit eines Sehnellessigbildners, der nach fünfzehnjährigem (iebra uch geöfnet wurde. Besonders bemerkenswert war diss starke Anwachsen des Stickstoffgehaltes der Späne. C. K i p pen ber qe r ${ }^{120}$ ) schildert die Fubrikationsverhältnisse des $W$ e i n ess i gs, die dazu führen, da $B$ Fssig, bei dessen Brereitung mindestens 20\%' Wein verwendet wurden. als Weinessig anzuerkennen ist. A. S j l v r m a $\mathrm{n}^{127}$ ) will die $\mathrm{K}$ on . trolle der fissigzusammensetzung durch Gefrierpunktwbestimmung durchiführen. Nach H. F i n k e 128) können G ̈̈r ungess i und Essenzessig durch Bestimmung des Ameisen. säuregehaltes unterschieden werden. Letzterer hat immer sehr viel höheren Gehalt an Ameisensäure als ersterer. Derselles Vf. 129) berichtet ïber V e r . wendung vergällten Branntweines z u r ks igfabrikation. Diese verrät sich durch Pyridingegenwart in Essig. Aus Essigsäureund Pyridingehalt läßt sich annähernd berechnen, wieviel vergällter Branntwein verwendet wurde.

H. Wüstenfeld, H. R $R^{+} B m$ an n und Th. $F \ddot{o h ~ r ~}{ }^{130}$ ) haben weitere Versuche zum A b.

121) D. R. P. 233 209; diese Z. 24, 959 (1911).

122 ) D. R. P. 239 530; diese 7. 25, 188 (1912).

123) D. R. P. 240473.

124) Deutsche Essigind. 15. 253.

125) Ibid. 15, 334

126) Diese Z. 24, 2006 (1911).

127) Chem.-Ztg. 35, 43; diese Z. 24, 996 (1911).

128) Deutsche Eusigind. 15, 145.

129) Ibid. 15, 113: diese Z. 24, 1491 (1911).

130) Ibid. 15. 387 . töten der Es a i g lo hen angestellt. Die bereits bekannte Wirkung des Chlornatriums läBt sich durch Kohlensäure erhöhen, auch Calcium- und Magnesiumsuperoxyd wirken tödlich.

[A. 68.]

\section{Über die Luminescenzanalyse, eine neue Untersuchungsmethode für Che- mikalien im ultravioletten Licht.}

(Mit Demonstrationen.)

Von Dr. H. Lehmaxs, Jena.

Vortrag in der Sitzung 1) des Markischen Bezirkspereing des Vereins deutscher Chemiker in Berlin am 27. Februar 1912; 2) des Bezirtyvereins Sarhsen. Thilringen des Vereins deutgcher Chemiker im Huttenmknnischen Inatitul dev Bergaksdemie zu F'reiberg am 10. Mnrz 1912

(Eingeg. 25.18. 1912)

M. H.! Ich habe die Fhre und das Vergnügen, Ihnen einen Apparat vorführen zu können, der eint" Bestrahlung mit intensivem. reinem ultravioletten Licht gestuttet, und der neuerdings von dem Zeibwerk in .Jena hergestellt wird. Apparate, wolche intensives ultravioletter Ijicht ausstrahlen, sind ja längst bekannt, ich erinnere hier nur an die Uviollampe von Solotet in Jena oder an die Quar\%. lampe von Heraeus. Djese Jampen ergeben zwar, wje kesagt, ein sehr intensives ultraviolettes Licht, aber dieses licht ist noch gemischt mit den sichtbaren Strahlen, so daß man die Wirkung der ultrnvioletten Struhlen allein nicht beobachten kann. Fs ist also noch eine Vorrichtung nötig. welehe d e sichtbaren sitrahlen von den ult raviolet ten scheidet. Derartige Vorrichtungen sind ja auch schom bekannt. Man kann diese Scheidung von Strahlen entweder durch Dispersion oder durch Absorption bewerkstelligen, z. B. durch Zerlegung des gemischten Lichtes durch ein l'risma, welches die strahlen bo. kanntlich zu einem Spektrum ausbreitet. Man braucht dann nur die sicht baren Strahlen in diesem Spektrum abzublenden und nur die ultravioletten zu benutzen. Aber derartige Forrichtungen haben für die Zwecke, welche ich Ihnen hier erklären will, große Nuchteila: einmal wird die Helligkeit durch solche Vorrichtungen stark herabgesetzt, und dann ist auch (ine geringe Beimischung von sichtbaren Strahlen seliwer zu vermeiden. Weit einfacher und zweckmäBiger ist für unsere Zwecke die Anwendung der Absorption, deren man sich bei der Verwendung von sog. Lichtfiltern bedient. Lichtfilter, die ein bestimmtes Gebiet in sichtbaren Spektrum her. ausschneiden oder gewissermaßen heraussieben, sind ja zur Genüge bekannt. Fs gibt l'latten, welche nur das rote Jicht durchlassen, solche, die nur das grüne durchlassen usw. Hiernach liegt es auf der Hand, daB auch Lichtfilter denkbar aind, die nur ultraviolettes Licht hindurchlassen. Auch derartige Lichtfilter sind bereits bekannt geworden, so hat z. B. l'rof. S' t $r$ a u b e l cine dünne Silber. fläche vorgeschlagen, welche nur ein enge's Spektralgebiet bei etwa $320 \% \mu$ hindurchläBt. Dieses Filter ist für astro-physikalische Zwecke wiederholt in Anwendung gekommen. Fïr unsere Zwecke reicht seine Durchlässigkeit jedoch nicht aus. Ferner hat der amerikanische Physiker Wood 